\title{
Review
}

Hua Feng*

\section{Research progress in thecorrelation between oral and stomach Hpinfections}

DOI: $10.2478 / \mathrm{ii}-2018-0021$

Received May 02, 2018; accepted May 18, 2018; published online June 29, 2018

\begin{abstract}
Helicobacter pylori (Hp) is one of the most common human pathogens. The infection caused by this bacterium is closely related to various clinical diseases. However, the eradication rate of conventional quadruple therapy has decreased, but the recurrence rate of infections has increased. The oral cavity is another major storage of $\mathrm{Hp}$ in addition to the stomach, and a certain homology exists between $\mathrm{Hp}$ in the oral cavity and $\mathrm{Hp}$ in the stomach. Periodontal treatment has a certain role in the killing of oral Hp, which can increase the eradication rate and reduce the recurrence rate of $\mathrm{Hp}$ in the stomach. This article summarizes the influence of oral $\mathrm{Hp}$ on the eradication rate and recurrence rate of gastric $\mathrm{Hp}$.
\end{abstract}

Keywords: Helicobacter pylori, oral cavity, eradication rate, recurrence rate

Helicobacter pylori (Hp) are infectious bacteria that colonize the stomach and duodenum. This species presents a high rate of infection [1] and can cause chronic inflammation of the stomach and duodenum. Hp infection can cause gastric mucosal damage, leading to chronic gastritis, gastric ulcer, and duodenal ulcer. More serious cases can lead to gastric cancer and gastric mucosa-associated lymphoid tissue lymphoma and other diseases. Hp gastritis is the most important risk factor for gastric cancer and peptic ulcer and its complications. The fourth Kyoto Consensus Conference showed that Hp gastritis should be defined as infectious gastritis, even if the patient is asymptomatic and does not have complications, such as peptic ulcer and gastric cancer. Hp is a carcinogen that causes gastric cancer. This mechanism was internationally recognized by the International Cancer Research Institute in 1994. Hp has been increasingly investigated since 1983, because Warren discovered Hp from the stomachs of patients with chronic gastritis. In 1989, Krajden et al. [2] noticed the importance of dental plaque in the study of patients with chronic gastritis and successfully developed $\mathrm{Hp}$ from the dental plaque. International studies have successively used immunological assays, polymerase chain reaction (PCR), and bacterial cultures to investigate and prove the presence of $\mathrm{Hp}$ infection in the oral cavity of patients with Hp gastritis [3]. A certain relationship was found between $\mathrm{Hp}$ in the stomach and that in the oral cavity. The oral cavity may be another region where $\mathrm{Hp}$ survives, and the oral infection of $\mathrm{Hp}$ is a susceptibility factor of periodontal disease. Several studies have confirmed that $\mathrm{Hp}$ is also related to several vascular diseases outside the digestive system, such as idiopathic thrombocytopenic purpura, atherosclerosis, ischemic heart disease, ischemic stroke, irondeficiency anemia, and central serous chorioretinopathy. In recent years, the eradication rate of gastric Hp has been decreasing, but the recurrence rate has been increasing. The recurrence of gastroduodenal lesions caused by Hp infection and the reinfection of Hp pose a great challenge for doctors and patients. This article reviews the latest advances in the study of the eradication and recurrence rates of stomach Hp infections and oral Hp infections both domestically and internationally. This article enables improved oral Hp detection in patients with Hp-associated gastrointestinal diseases and proves the necessity and feasibility of routine eradication of stomach Hp infections combined with oral Hp infections. Thus, new prospects for Hp-related research are expected.

Department of Emergency Medicine, Qinzhou TCM Hospital, Qingzhou, China

*Correspondence: Hua Feng, E-mail: huafqz@sina.com 


\section{Introduction to $\mathrm{Hp}$}

$\mathrm{Hp}$ is a Gram-negative bacterium with unipolar, multi-flagellate, obtuse-end, and spiral-curved shapes. It is microaerobic with an environmental oxygen requirement of $5 \%-8 \%$. It is mainly parasitized in the nearby mucosa of stomach pylorus and gastric antrum. It is infectious and can cause abdominal pain, bloating, upper abdominal pain, discomfort, hunger, and even vomiting and other upper gastrointestinal symptoms. Hp in gastric antrum is also related to the occurrence of gastric ulcer [4]. Severe cases can also cause the occurrence of gastric cancer [5]. Several data have shown that Hp infection has obvious regional differences and is closely related to economic conditions, public health conditions, and living habits. The infection rate of $\mathrm{Hp}$ and the incidence of stomach diseases obviously vary in countries with different development conditions. The better the country's economic conditions, the lower the Hp infection rate and the incidence of stomach diseases [6]. The results of inspections in healthy people showed that the detection rate in developing countries was approximately 50\%-70\%, while that in developed countries was generally lower than $30 \%$. The examination results in patients with gastritis and gastric ulcer showed that the detection rate in developing countries was $80 \%-100 \%$ [7]. With detection rate of $\mathrm{Hp}$ as high as $61 \%$, China, as a developing country, is an area with high Hp infection [8]. Kadayifci et al. [9] showed that the eradication rate of gastric Hp had decreased significantly since 2000 and the recurrence rate in developing countries is higher than that in developed countries.

\section{Shared homology of gastric Hp with oral Hp}

Epidemiological surveys showed that the Hp-positive rate of households without separate meals was significantly higher than that of households with separate meals [10]. Zou and Li [11] divided the patients into two groups, with group A as gastric Hp-positive patients and group B as gastric Hp-negative patients. The same method was used to detect oral $\mathrm{Hp}$ in the two groups, and the results showed that the oral positive rate was higher in group A than in group B. This result indicated that oral Hp and stomach Hp had certain correlation. Assumpção et al. [12] studied a total of 99 patients with dyspepsia and used PCR to compare and analyze Hp in the oral cavity and gastric mucosa of the same patient. The Hp genotype in the oral cavity was found to be consistent with that in the gastric mucosa in $89 \%$ of patients. Wang et al. [13] used PCR to detect four different Hp genotypes in the dental plaque and gastric mucosa of 31 intragastric and oral Hp-positive patients. The four genotypes were cavA, vacAm1, vacAm2, and vacAs1. The results showed that $95 \%$ of at least one genotype in the dental plaque was consistent with that in the gastric mucosa and $27 \%$ of all genotypes in the dental plaque were consistent with those in the gastric mucosa. Wan et al. [14] used fluorescence quantitative-PCR, (FQ-PCR) to analyze 27 patients who failed gastric Hp eradication, of which 25 patients were found to be oral Hp positive and were analyzed using single-strand conformation polymorphism (SSCP). The results showed that 19 patients had the same SPCP banding pattern, while the remaining six cases had at least one same banding type. Lukes et al. [15] used PCR to detect the genotypes of Hp in the oral cavity and stomach of the same patient. The results showed that the genotypes of $\mathrm{Hp}$ in the oral cavity and stomach were different, which may have been caused by the homology between gastric Hp and oral Hp of the same patient. Although several experiments have shown that the gastric Hp and oral Hp have different genotypes, numerous experiments have also demonstrated contrasting results. The problem of homology remains controversial.

\section{Eradication of oral Hp infection}

Tursi et al. [16] found that $\mathrm{Hp}$ is mainly transmitted through feces to mouth, mouth to mouth, stomach to mouth, stomach to stomach, and examination and treatment equipment, among others. The oral cavity is one of the pathways for Hp to enter the digestive tract. Oral Hp can not only cause oral cavity diseases but also has certain effects on the treatment of Hp-associated gastropathy. Thus, whether the treatment is 
Hp-related gastric diseases or Hp-related oral diseases, the prompt discovery and eradication of Hp in the oral cavity is very important. Socransky et al. [17] found that when eradicating Hp from patients with positive gastric and oral $\mathrm{Hp}$, the standard triple therapy had poor oral $\mathrm{Hp}$ eradication rate but a good effect on the eradication of gastric Hp. Gebara et al. [3] treated 30 patients with positive oral Hp and gastric Hp using standard triple therapy. At 3 months after 7 days of treatment, the oral $\mathrm{Hp}$ and gastric Hp were measured by the same method. The results showed that the positive rate of oral $\mathrm{Hp}$ was $60 \%$, while that of gastric $\mathrm{Hp}$ was $10 \%$. The eradication rate of gastric Hp in the stomach was significantly higher than that of oral Hp. This experiment proved that the standard triple therapy had poor effect on the eradication of oral Hp. Numerous studies have shown that Hp in the dental plaque was difficult to remove by only medical treatment [3]. However, oral scaling can effectively remove supraorbital and subgingival plaques and reduce the attachment of Hp. Hp is present in not only supraorbital and subgingival plaques but also the saliva, gingival crevicular fluid, and tongue coating. After the periodontal scaling, residual Hp in the oral cavity is likely to be reattached to the periodontal pocket or newly formed dental plaque and then enters into the stomach through the diet or other routes.

\section{Influence of oral $\mathrm{Hp}$ on the eradication rate of gastric $\mathrm{Hp}$}

Song and Li [18] classified patients with positive gastric Hp into two groups. One group underwent eradication therapy with periodontal treatment and mouthwash. By contrast, the other group did not undergo periodontal treatment and mouthwash was not used. The results showed that the eradication rate of gastric Hp was higher in patients who received periodontal therapy and used mouthwash. Miyabayashi et al. [19] divided patients into groups A and B. Group A included patients with positive gastric and oral $\mathrm{Hp}$ at the same time, while group B consisted of gastric Hp-positive and oral Hp-negative patients. The two groups had the same eradication therapy of $\mathrm{Hp}$. The results demonstrated that the eradication rate of group A was $52.1 \%$, while that of group B was $91.6 \%$. At follow-up after 2 years, the eradication rate in group A was $69.5 \%$, while that in group B was 95.8\%, and the difference was statistically significant. Lou et al. [20] studied 81 patients with positive gastric and oral $\mathrm{Hp}$. The patients were randomly divided into groups A and B. Group A was administered quadruple therapy, while group B was administered quadruple therapy+DiBice gargle treatment. The oral Hp and gastric Hp were reexamined after 4 weeks of treatment. The results showed that the eradication rate of gastric Hp was significantly lower in group A $(60.98 \%)$ than in group B $(90.00 \%)$ $(P<0.05)$. The results also showed that the eradication rate of oral Hp in group A $(17.7 \%)$ was lower than that in group $\mathrm{B}(65.0 \%)(P<0.05)$. Thus, oral $\mathrm{Hp}$ was found to have a certain effect on the eradication of gastric Hp, especially for long-term efficacy. Killing oral $\mathrm{Hp}$ can significantly increase the eradication rate of gastric Hp. Meanwhile, Namiot et al. [21] found that daily adherence to brushing teeth and maintaining mouth hygiene with mouthwash after meal had no significant effect on the eradication rate of gastric Hp. This study showed that the eradication rate of Hp cannot be improved by relying solely on oral hygiene. Thus, a few studies on whether eradication of oral $\mathrm{Hp}$ can reduce the eradication rate of gastric $\mathrm{Hp}$ have been conducted. Additional evidence is needed to reach a more conclusive report.

\section{Influence of oral $\mathrm{Hp}$ on the recurrence rate of gastric $\mathrm{Hp}$}

Conventional quadruple therapy, i.e., proton pump inhibitors, colloidal pectin, and two kinds of biological factors, is currently the most commonly used and most effective treatment to eradicate Hp. However, in recent years, the recurrence rate of the disease has increased. This phenomenon may be due to the fact that conventional quadruple therapy has no effect on the eradication of oral $\mathrm{Hp}$ or Hp persists in the dental plaque, gingival crevicular fluid, and saliva after treatment and then is swallowed into the stomach. Jia et al. [22] randomly divided the gastric Hp eradication patients into three groups. Group A focused on oral hygiene and the number of teeth brushed and used mouthwash. Group B used the method of group A+oral scaling, 
while group C, as the control group, did not do any treatment. After 6 months, the same method was used to detect the recurrence rate of gastric $\mathrm{Hp}$ in each group, and the results showed that the relapse rate was significantly lower in group A than in group B and the relapse rate of group C was significantly higher than that of the other two groups. Thus, daily plaque control, ultrasonic scaling, and manual subgingival scaling to control dental plaque can significantly reduce the recurrence rate of $\mathrm{Hp}$ in the stomach. Bouziane et al. [23] studied the influence of oral scaling on the eradication and recurrence rates of gastric Hp and comprehensively analyzed data collected from studies prior to 2012. The results showed that oral scaling could reduce the recurrence rate of gastric $\mathrm{Hp}$. These studies demonstrated that after eradication therapy of gastric Hp, Hp remained in the oral cavity, saliva, and dental plaque and the retained Hp could cause the recurrence of gastric Hp. Therefore, the eradication of oral $\mathrm{Hp}$ is the basic measure to increase the eradication rate and reduce the recurrence rate of gastric Hp [24].

\section{Prospects}

The prevention of reinfection or recurrence after eradication of Hp has been an important topic worldwide. At present, the recurrence of $\mathrm{Hp}$ may be caused by the reinfection of new strains or the original strain being not completely cleared and rekindled [25]. Studies have shown that given the absence of medicines that can eradicate $\mathrm{Hp}$ from the oral cavity, drugs are hardly effective, especially when encountering plaque-forming microorganisms with a "biofilm" structure [26]. Given the difficulty in eradicating oral Hp, this condition can lead to the recurrence of $\mathrm{Hp}$ in the stomach, which in turn causes the recurrence of gastrointestinal diseases [27]. Clinical attention is only focused on eradicating gastric Hp without paying attention to the influence of oral $\mathrm{Hp}$ infection on the patients. This practice has resulted in the very high recurrence rate of gastric Hp. Therefore, the eradication and prevention of oral Hp should be paid attention to maximize the eradication rate and reduce the recurrence rate of gastric $\mathrm{Hp}$. In conclusion, the key to successful eradication of gastric $\mathrm{Hp}$ is the ability to completely remove oral Hp. Focusing on the therapy of oral Hp can not only increase the eradication rate and reduce the recurrence rate of gastric $\mathrm{Hp}$ but can also play a protective role in oral care.

Acknowledgments: None.

Conflict of interest: The author states no conflicts of interest.

\section{References}

[1] Tu B., Ji W., Hao Z., Lu Y., Zhu W., Survey of demand for oral preventive services among preschool children in Pudong new area, Prac. Prev. Med., 2014, 21(1), 1664-1666.

[2] Krajden S., Fuksa M., Anderson J., Kempston J., Boccia A., Petrea C., et al. Examination of human stomach biopsies, saliva, and dental plaque for Campylobacter pylori, J. Clin. Microbiol., 1989, 27(6),1397-1398.

[3] Gebara E.C., Faria C.M., Pannuti C., Chehter L., Mayer M.P., Lima L.A., Persistence of Helicobacter pylori in the oral cavity after systemic eradication therapy, J. Clin. Periodontol., 2006, 33, 329-333.

[4] Forman D., Newell D.G., Fullerton F., Yarnell JW, Stacey AR, Wald N, et al., Association between infection with Helicobacter pylori and risk gastric cancer: evidence from a prospective investigation, BMJ, 1991, 302, 1302-1305.

[5] Lee A., Fox J., Hazell S., Pathogenicity of Helicobacter pylori: a perspective, Infect. Immun., 1993, 61, 1601-1610.

[6] Banatvala N., Clements L., Abdi Y., Graham JY, Hardie JM, Feldman RA, Migration and Helicobacter pylori seroprevalence: Bangladeshi migrants in the UK, J. Infect., 1995, 31, 133-135.

[7] Megraud F., Epidemiology of Helicobacter pylori infection, Gastroenterol. Clin. North Am., 1993, 22, 73-88.

[8] Li Y., Hu P., Du G., Hazell S.L., The prevalence of Helicobacter pylori infection in the People's Republic of China, Am. J. Gastroenterol., 1991, 86, 446-449.

[9] Kadayifci A., Buyukhatipoglu H., Cemil Savas M, Simsek I., Eradication of Helicobacter pylori with triple therapy: an epidemiologic analysis of trends in Turkey over 10 years, Clin. Ther., 2006, 28(11), 1960-1966. 
[10] Chow T.K., Lambert J.R., Wahlqvist M.L., Hsu-Hage B.H., Helicobacter pylori in Melbourne Chinese immigrants: evidence for oral-oral transmission via chopsticks, J. Gastroenterol. Hepatol., 1995, 10, 562-569.

[11] Zou Q., Li R., Helicobacter pylori in the oral cavity and gastric mucosa a meta-analysis, J. Oral Pathol. Med., 2011, 40, 317-324.

[12] Assumpção M.B., Martins L.C., Melo Barbosa HP, Barile K.A., de Almeida SS, Assumpção P.P., et al., Helicobacter pylori in dental plaque and stomach of patients from Northern Brazil, World J. Gastroenterol., 2010, 16(24), 3033-3039.

[13] Wang J., Chi D., Laffan J.J., Li C., Ferguson DA Jr, Litchfield P., et al., Comparison of cytotoxin genotypes of Helicobacter pylori in stomach and saliva, Dig. Dis. Sci., 2002, 47(8), 1850-1856.

[14] Wan Y., Chen X., Zhao P., Wang S., Study on the relationship between Hp infection in oral cavity and stomach, Int. J. Lab. Med., 2016, 37(18), 2566-2570.

[15] Lukes P., Pavlik E., Potuznikova B., Plzák J., Nártová E., Doseděl J., et al., Comparison of Helicobacter pylori genotypes obtained from the oropharynx and stomach of the same individuals - a pilot study, Prague. Med. Rep., 2012, 113(3), 231-239.

[16] Tursi A., Cammarota G., Papa A., Cuoco L, Gentiloni N, Fedeli P, et al., The modes of transmission of Helicobacter pylori infection, Recenti. Prog. Med., 1997, 88(5), 232-236.

[17] Socransky S.S., Haffajee A.D., Cugini M.A., Smith C., Kent RL Jr, Microbial complexes in subgingival plaque, J. Clin. Periodontol., 1998, 25(2), 134-144.

[18] Song H.Y., Li Y., Can eradication rate of gastric Helicobacter pylori be improved by killing oral Helicobacter pylori, World J. Gastroenterol., 2013, 19(39), 6645-6650.

[19] Miyabayashi H., Furihata K., Shimizu T., Ueno I., Akamatsu T., Influence of oral Helicobacter pylori on the success of eradication therapy against gastric Helicobacter pylori, Helicobacter, 2000, 5(1), 30-37.

[20] Lou X., Shen L., Chen Z., Li L., Correlation between oral Hp infection and eradication rate of gastric Hp infection, World Chin. J. Digestol., 2016, 24(18), 2918-2922.

[21] Namiot D.B., Namiot Z., Kemona A., Bucki R., Gotebiewska M., Oral health status and oral hygiene practices of patients with peptic ulcer and how these affect Helicobacter pylori eradication from the stomach, Helicobacter, 2007, 12(1), 63-67.

[22] Jia C., Jiang G., Yang X., Influence of two kinds of dental plaque control methods on gastric mucosal reinfection of Hp, West China J. Stomatol., 2009, 2(27), 172-174.

[23] Bouziane A., Ahid S., Abouqal R. Ennibi 0 Effect of periodontal therapy on prevention of gastric Helicobacter pylori recurrence: a systematic review and meta-analysis, J. Clin. Periodontol., 2012, 39(12), 1166-1173.

[24] Ye G., Treatment strategy of oral Hp infection - an important problem that must be faced, Chin. J. Med., 2012, 92(10), 659-661.

[25] Niv Y., H. pylori recurrence after successful eradication, World J. Gastroenterol., 2008, 14(10), 1477-1478.

[26] Broutet N., Tchamgoue S., Pereira E. Lamouliatte H, Salamon R, Mégraud F, Risk factors for failure of Helicobacter pylori therapy-results of an individual data analysis data analysis of 2751 patients, Aliment. Pharmacol. Ther., 2009, 17(1), 99-109.

[27] Czesnikiewicz G.M., Loster B., Bielanski W., Guzik TJ, Konturek PC, Zapala J, et al., Implications of oral Helicobacter pylori for the outcome of its gastric e-radication therapy, J. Clin. Gastroentenol., 2007, 41(2), 145-151. 